\title{
Diets Rich in Olive Oil, Palm Oil, or Lard Alter Mitochondrial Biogenesis and Mitochondrial Membrane Composition in Rat Liver
}

\author{
Youzan Ferdiand Djohan $\mathbb{D}^{1},{ }^{1}$ Massara Camara-Cissé, ${ }^{1}$ Gilles Fouret, ${ }^{2}$ Béatrice Bonafos, ${ }^{2}$ \\ Bernard Jover, ${ }^{3}$ Jean-Paul Cristol, ${ }^{4}$ Charles Coudray, ${ }^{2}$ Christine Feillet-Coudray, ${ }^{2}$ \\ and Eric Badia ${ }^{3}$ \\ ${ }^{1}$ Laboratoire de Biochimie CHU, Université Félix Houphouët-Boigny, Cocody, Abidjan, Côte d'Ivoire \\ ${ }^{2}$ DMEM, INRA, Université Montpellier, Montpellier, France \\ ${ }^{3}$ PhyMedExp, Université Montpellier, INSERM U1046, CNRS, UMR 9214, Montpellier, France \\ ${ }^{4}$ Laboratoire de Biochimie, CHU-Lapeyronie, Montpellier, France
}

Correspondence should be addressed to Youzan Ferdiand Djohan; djohanferdinand@gmail.com

Received 23 October 2021; Revised 16 January 2022; Accepted 28 January 2022; Published 21 February 2022

Academic Editor: Néstor Gutiérrez-Méndez

\begin{abstract}
Copyright (C) 2022 Youzan Ferdiand Djohan et al. This is an open access article distributed under the Creative Commons Attribution License, which permits unrestricted use, distribution, and reproduction in any medium, provided the original work is properly cited.
\end{abstract}

Palm oil (crude or refined) and lard are rich in SFA, while olive oil is rich in polyunsaturated fatty acids. SFA are considered harmful to health, while polyunsaturated fatty acids are beneficial to health. The aim of this study was to determine the effect of diets rich in crude PO, refined PO, OO, or lard on the mitochondrial membrane, the activity of mitochondrial respiratory chain complexes, and mitochondrial biogenesis. This was an experimental study in male Wistar rats fed a diet containing $30 \%$ of each oil. Rats had free access to food and water. After being fed for 12 weeks, animals were sacrificed and liver mitochondria were collected. This collection was used to determine membrane potential and ROS production, membrane phospholipid and fatty acid composition, citrate synthase activity and respiratory chain complex, cardiolipin synthase protein expression, and expression of selected genes involved in mitochondrial biogenesis. We found that diets rich in olive oil, palm oil, or lard altered mitochondrial biogenesis by significantly decreasing Pgc1 $\alpha$ gene expression and altered the fatty acid composition of rat liver mitochondrial membrane PL.

\section{Introduction}

The most important role of mitochondria is the production of adenosine triphosphate (ATP) in the cell, which is used as an energy source. In addition to their role in cellular energy metabolism, mitochondria are also involved in cell signalling, differentiation and death, and in the control of the cell cycle and cell growth [1]. These processes in turn influence mitochondrial biogenesis $[2,3]$. Their dysfunction has been associated with several human diseases [4]. The inner and outer mitochondrial membranes are made up of phospholipids (PL) [5]. The fatty acid composition of PL is diet dependent and influences mitochondrial membrane functions [6]. Insulin resistance (IR) alters mitochondrial function through (i) decreased expression of the gene encoding peroxisome proliferator activator receptor $\gamma$ coactivator- $1 \alpha \quad(\mathrm{PGC} 1 \alpha)$ with decreased mitochondrial density [7], (ii) decreased oxidative phosphorylation [8], and (iii) abnormalities in mitochondrial morphology and impaired mitochondrial respiration and ATP synthesis [8].

High-fat diets (HFD) that favor their accumulation in the form of diacylglycerol, triacylglycerol, and ceramides are directly associated with IR $[9,10]$. In addition, studies have highlighted the role of saturated fatty acids (SFA), particularly palmitic acid, in the development of IR $[11,12]$. It should be noted that the majority of studies on fatty acids 
(saturated or unsaturated) use cafeteria diets, i.e., composed of a mixture of fatty acids. The particularity of this study lies in the fact that it uses vegetable oils with all their components (SFA, MUFA, PUFA, vitamins, polyphenols, etc.). Palm oil (PO) is rich in SFA, which represent about $50 \%$ of total fatty acids $[13,14]$. SFA content of PO is close to that of lard, which is composed of $45 \%$ SFA [15]. Olive oil (OO) is very rich in unsaturated fatty acids [16] and polyphenols [17]. These properties give it nutritional benefits $[18,19]$.

However, it is not known whether excess consumption of crude PO, refined PO, OO, or lard alters the physicochemical properties of the mitochondrial membrane, the activity of mitochondrial respiratory chain complexes, and mitochondrial biogenesis.

The aim of this study was to determine the effect of diets rich in crude $\mathrm{PO}$, refined $\mathrm{PO}, \mathrm{OO}$, or lard on the mitochondrial membrane, the activity of mitochondrial respiratory chain complexes, and mitochondrial biogenesis.

\section{Materials and Methods}

2.1. Animals and Diets. Forty young male Wistar rats (Charles River, L'Arbresle, France) aged 6 weeks at the beginning of experiments were housed under conditions of constant temperature $\left(20-22^{\circ} \mathrm{C}\right)$, humidity $(45-50 \%)$, and a standard dark cycle (20.00-08.00 h) with free access to food and water. Rats were randomised into five groups of eight animals and fed for 12 weeks either a standard rat chow diet (control diet) or one of the four HFD. In control diet, 11\% of the energy was given by fat ( $5 \%$ soybean oil), whereas in HFD, 56\% of the energy was provided by fat intake [20]. The fat-enriched diets consisted of $2.5 \%(\mathrm{w} / \mathrm{w})$ of soybean oil and $30 \%(w / w)$ of crude PO, refined PO (Sania Cie, Abidjan, Côte d'Ivoire), OO (virgin olive oil, bought in a supermarket), or lard (Alva, Rezé, France). The detailed composition of these experimental diets is given in Table 1. After being fed for 12 weeks, rats were anaesthetized with $1 \%$ pentobarbital $(50 \mathrm{mg} / \mathrm{kg}$ ) (Ceva Santé Animale, Libourne, France).

The experiments complied with the guidelines for the care and use of laboratory animals (National Academies Press (US), 8th edition, 2011), and all procedures were approved by the local ethical committee (reference CEEALR-12002, Montpellier, France).

2.2. Rat Sacrifice and Sampling. Rats were anaesthetized with $1 \%$ pentobarbital (50 mg/kg ip) (Ceva Santé Animale, Libourne, France). The rat liver was quickly removed, rinsed in $0.9 \% \mathrm{NaCl}$ solution, cut into different parts that were immediately frozen in liquid nitrogen, and then kept at $-80^{\circ} \mathrm{C}$ until analysis, except one part that was used for the immediate isolation of fresh mitochondria.

Mitochondria were isolated from the fresh liver by the differential centrifugation techniques previously described [21]. One aliquot of the mitochondrial suspension was used for mitochondrial membrane potential and reactive oxygen species (ROS) production. One aliquot was frozen at $-80^{\circ} \mathrm{C}$ for other measurement. Protein content was determined
TABLE 1: Diet composition $(\mathrm{g} / \mathrm{kg})$ on the basis of the AIN-93M diet formulation.

\begin{tabular}{lccccc}
\hline Ingredients & Control & $\begin{array}{c}\text { Crude } \\
\text { PO }\end{array}$ & $\begin{array}{c}\text { Refined } \\
\text { PO }\end{array}$ & OO & Lard \\
\hline Casein & 165 & 200 & 200 & 200 & 200 \\
Cornstarch & 443 & 234 & 234 & 234 & 234 \\
Maltodextrin & 144 & 80 & 80 & 80 & 80 \\
Sucrose & 100 & 53 & 53 & 53 & 53 \\
Soybean oil & 50 & 25 & 25 & 25 & 25 \\
Crude palm oil & 0 & 300 & 0 & 0 & 0 \\
Refined palm oil & 0 & 0 & 300 & 0 & 0 \\
Olive oil & 0 & 0 & 0 & 300 & 0 \\
Lard & 0 & 0 & 0 & 0 & 300 \\
Cellulose & 50 & 50 & 50 & 50 & 50 \\
Mineral mix (AIN- & 35 & 42 & 42 & 42 & 42 \\
93M) & & & & & \\
Vitamin mix (AIN- & 10 & 12 & 12 & 12 & 12 \\
93M) & 2 & 2.4 & 2.4 & 2.4 & 2.4 \\
L-cystine & 1.5 & 1.8 & 1.8 & 1.8 & 1.8 \\
Choline chloride & 1000 & 1000 & 1000 & 1000 & 1000 \\
Total & & & & & \\
\hline
\end{tabular}

according to Bradford with bovine serum albumin as the standard [22].

2.3. Liver Triglycerides and Histological Lipid Analyses. Liver samples were homogenized in $\mathrm{NaCl}(9 \mathrm{~g} / \mathrm{L})$ and Triton $\mathrm{X}-100(0.1 \%)$. The liver contents of triglycerides were quantified on the liver homogenates by enzymatic methods using the triglycerides PAP kit (bioMérieux, France).

To visualise hepatic lipid accumulation, Oil Red O stainings were applied. Frozen liver $5 \mu \mathrm{m}$ sections were stained with Oil Red O. Liver sections were observed under an AxioImager Z1 Zeiss microscope. Images were acquired using the AxioImager software driving a CoolSNAP CCD camera on 5 fields (20X) per section. The staining area was measured in a given field and expressed as a percentage of the total area within the field. For each group, liver samples from 6 to 8 rats were prepared and stained.

\subsection{Liver Mitochondrial Membrane Characteristics and En-} zyme Activities. Liver mitochondrial membrane potential and ROS production were assessed as previously described $[23,24]$. The enzymatic activity of citrate synthase (CS) in the liver and mitochondrial respiratory complexes (complex I, complex II + III, and complex IV) in liver mitochondria were determined spectrophotometrically as previously described [25]. In brief, the activity of CS was measured by following the color of 5-thio-2-nitrobenzoic acid, which is generated from 5,5'-dithiobis-2-nitrobenzoic acid present in the reaction of citrate synthesis, and caused by the deacetylation of acetyl-CoA. Complex I activity was measured spectrophotometrically at $600 \mathrm{~nm}$ during $45 \mathrm{~s}$ by following the reduction of 2,6-dichloroindophenol by electrons accepted from decylubiquinol, which itself reduced after oxidation of NADH by complex I [26]. Complex II activity was measured spectrophotometrically at $600 \mathrm{~nm}$ by following the reduction of 2,6-dichloroindophenol by the succinate during 
120 s. Complex II + III activities were measured spectrophotometrically by following the oxidation of cytochrome $\mathrm{c}$ at $550 \mathrm{~nm}$ during $90 \mathrm{~s}$ [27]. Cytochrome c oxidase (complex IV) activity was measured spectrophotometrically by following the oxidation of reduced cytochrome c at $550 \mathrm{~nm}$ during $30 \mathrm{~s}$ [28].

2.5. Analysis of Phospholipids in Liver Mitochondria. Mitochondrial membrane phospholipids were analysed as previously described [21]. Liver mitochondrial suspensions were extracted using a mixture of chloroform-methanol (2: $1 ; \mathrm{v} / \mathrm{v}$ ) according to Folch et al. [29] in the presence of $50 \mathrm{mg} / \mathrm{l}$ of butylated hydroxytoluene to prevent lipid oxidation. Phosphorus was quantified on Folch extracts of mitochondrial suspensions in order to determine total phospholipid quantity as previously described [21,30]. In brief, mitochondrial lipid extracts were automatically applied on silica gel 60 HPTLC plates $(250 \mu \mathrm{m}, 20 \times 10 \mathrm{~cm})$, pretreated with $2-3 \%(\mathrm{w} / \mathrm{v})$ boric acid in ethanol $(100 \%)$, on a $4 \mathrm{~mm}$ band width using the ATS4 apparatus (CAMAG). The lipid spots' development was performed with methanolacetic acid-pentane-chloroform $(15: 10: 30: 45$, by vol.), which allowed the separation of phospholipids and neutral lipids on a $60-\mathrm{mm}$ total migration distance. The scanning of the plates was carried out using a TLC scanner 3 (CAMAG) operating in the reflectance mode. The plates were scanned at $715 \mathrm{~nm}$ after dipping in a solution of Blue Spray (Sigma) (Blue Spray-4.2 $\mathrm{M} \mathrm{H}_{2} \mathrm{SO}_{4}$-acetone, $1: 2: 3$, by vol.) and heating for $3 \mathrm{~min}$ at $55^{\circ} \mathrm{C}$. The different classes of phospholipids (sphingomyelin $=\mathrm{SM}$, lysophosphatidylcholine $=$ LPC, $\quad$ phosphatidylcholine $=$ PC, phosphatidylinositol $=$ PI, phosphatidylserine $=$ PS, phosphatidylethanolamine $=\mathrm{PE}$, and cardiolipin $=\mathrm{CL}$ ) were finally identified by comparing their retention factor with authentic standards and quantified using calibration curves of the same standards.

Liver mitochondrial membrane phospholipid fatty acid analysis was determined as previously described [21, 31]. Fatty acid composition of mitochondrial phospholipids was compared in terms of the percentage content of various fatty acids.

2.6. Liver Lipid Mitochondrial Desaturase Indices and Unsaturation Index. Liver lipid mitochondrial desaturase indices and unsaturation index were determined as previously described [21]. In brief, desaturase indices are based on the ratio of product to precursor of individual fatty acids for a given desaturation reaction, so these indices were calculated as follows: desaturase $\Delta 9$ (16: $1 n-7 / 16: 0)$; desaturase $\Delta 6$ (18: $3 n-6 / 18: 2 n-6)$; and desaturase $\Delta 5(20: 4(n-6) / 20$ : $3(n-6))$ [32]. The unsaturation index (UI) was calculated from the relative percentage of each type of MUFA and PUFA multiplied by the number of double bonds present in the molecule [25].

2.7. Western Blotting Was Performed as Previously Described [33]. In brief, whole-cell protein lysates were prepared in the lysis buffer: $20 \mathrm{mM}$ Tris $\mathrm{pH}$ 8, $50 \mathrm{mM}$ DL-Dithiothreitol, $150 \mathrm{mM} \mathrm{NaCl}, 2 \mathrm{mM}$ EDTA, 1\% Triton X-100, 0.1\% SDS, $1 \mathrm{mM}$ PMSF, $1 \mathrm{mM}$ orthovanadate, and $1 \%(\mathrm{v} / \mathrm{v})$ of antiprotease cocktail (Sigma). Proteins were resolved by SDSPAGE and then transferred to nitrocellulose membranes using refrigerated Tris-glycine transfer buffer at $20 \mathrm{~V}$ overnight. Membranes were blocked in 5\% nonfat milk in PBS (without Tween) for $1 \mathrm{~h}$ at room temperature. Then, membranes were incubated overnight with primary antibody of CL synthase (CLS) (Abcam, France) and tubulin (Sigma-Aldrich, France) in blocking buffer. After washing with $\mathrm{PBS} /$ Tween under gentle agitation, membranes were incubated for $45 \mathrm{~min}$ in the dark with the fluorescent-labeled secondary antibodies and finally quantified using the Odyssey infrared imaging system (LI-COR, Lincoln, USA).

2.8. Liver $m R N A$ Expression. Real-time quantitative PCR (RT-qPCR) was used to measure the expression of several genes involved in liver mitochondrial biogenesis. Total RNA was extracted with TRIzol reagent (Invitrogen Life Technologies, Cergy Pontoise, France). Reverse transcription reactions were performed on $500 \mathrm{ng}$ total RNA using a reverse transcription Takara Kit (Takara Bio Europe, France). The mRNA expressions of target genes (mitochondrial transcription factor A (Tfam), peroxisome proliferator activator receptor $\gamma$ coactivator- $1 \alpha(\operatorname{Pgc} 1 \alpha)$, and nuclear respiratory factor-1 (Nrf1)) were determined by RTqPCR using the LightCycler ${ }^{\circledR} 480$ SYBR Green I Master (Roche Applied Science, France). The results were normalized to Rplp0 gene. The primer sequences used for realtime RT-PCR are the following: Rplp0 forward CACTGGCTGAAAAGGTCAAGG, Rplp0 reverse GACTTGGTGTGAGGGGCTTA; Pgcl $\alpha$ forward TGTGGAACTCTCTGGAACTGC, Pgcl $\alpha$ reverse GCCTTGAAAGGGTTATCTTGG; Tfam forward AGCTAAACACCCAGATGCAAA, Tfam reverse TCAGCTTTAAAATCCGCTTCA; Nrf1 forward TTATTCTGCTGTGGCTGATGG, Nrf1 reverse CCTCTGATGCTTGCGTCGTCT.

2.9. Statistical Analysis. The results were expressed as mean values $\pm \mathrm{SD}, n=7-8$ animals per group. Statistical analysis was based on one-way ANOVA followed by a Tukey-Kramer multiple comparisons test. When statistical variances were unequal, a Welch test was performed. The limit of statistical significance was set at $p<0.05$. The group mean values with different letters ( $a, b, c$, and d) are significantly different. Statistical analyses were performed using the StatView program (SAS Institute, Cary, NC, USA).

\section{Results and Discussion}

3.1. Hepatic Lipid Infiltration. All HFD promoted an increase in liver weight (crude $\mathrm{PO}+9 \%$, lard $+9 \%$, refined $\mathrm{PO}+22 \%$, and $\mathrm{OO}+16 \%)$. Compared to control diet, these increases were significant with refined $\mathrm{PO}$ and $\mathrm{OO}$ diets $(p=0.0301)$ (Figure 1(a)). However, high dietary intake of OO did significantly increase liver TG levels $(p<0.036)$, 


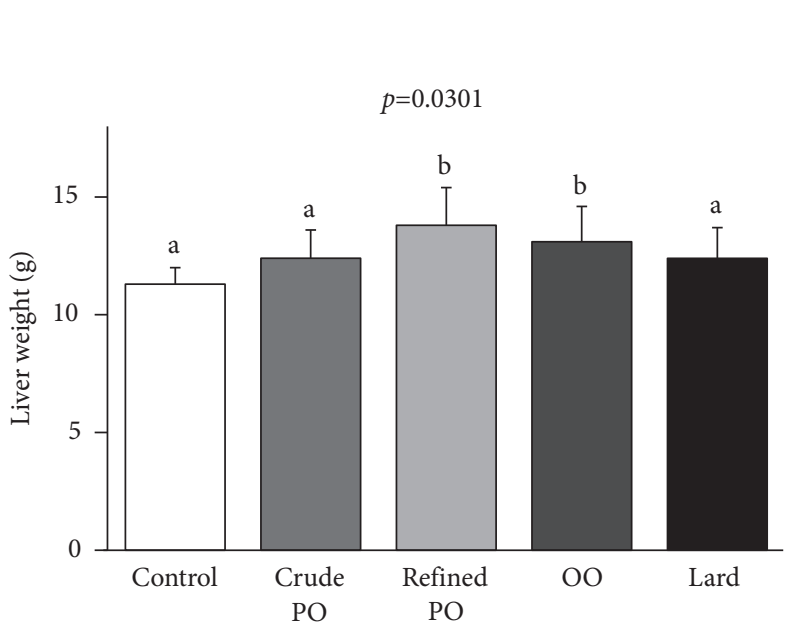

(a)

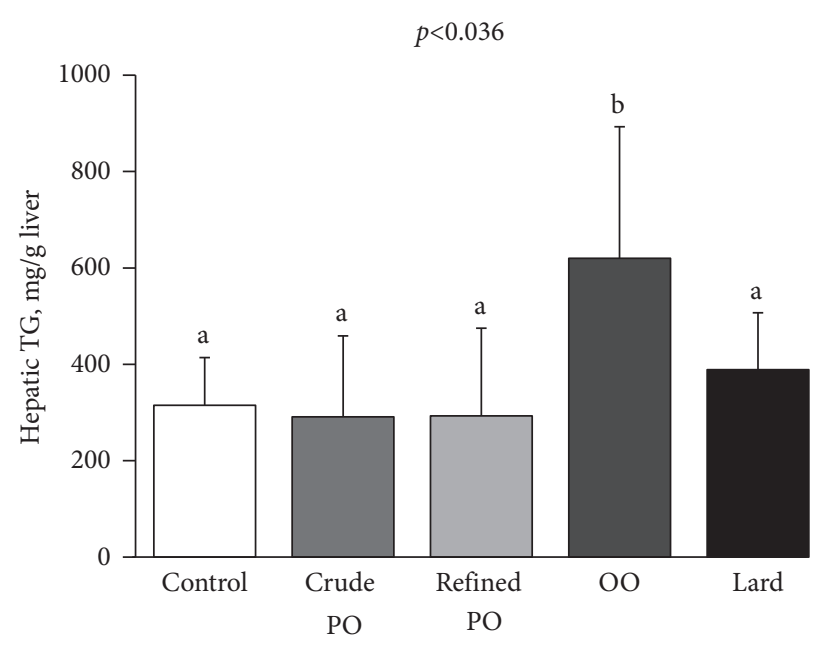

(b)

FIGURE 1: Effect of high dietary intake of palm oil, olive oil, and lard on (a) liver weight and (b) liver triglycerides. The results are expressed as mean values $\pm \mathrm{SD}, n=7-8$ animals per group. The limit of statistical significance was set at $p<0.05$. Bar graphs with different letters ( $\mathrm{a}$, $\mathrm{b}$, and c) are significantly different. OO, olive oil; PO, palm oil; TG, triglycerides.

whereas the high intake of both $\mathrm{PO}$ and lard did not (Figure 1(b)).

In addition to the high hepatic TG level, the liver histological lipid staining with Oil Red $\mathrm{O}$, reflecting hepatic steatosis, showed significant differences $(p<0.0281)$ among the studied groups (Figure 2). More specifically, compared to control diet, the high intake of OO did increase the liver lipid area, whereas the high intake of PO and lard did not.

The accumulation of hepatic lipid induced by the consumption of $\mathrm{OO}$ has been demonstrated in many studies. Arbones-Mainar et al. [34] found the same results in mice fed for 10 weeks with three HFD containing $20 \%$ fat. This study compared the effects of three vegetable oils (PO, low polyphenol $\mathrm{OO}$, and high polyphenol OO) on the liver. These authors observed a significantly greater hepatic steatosis with OO diets than with PO diet. Ferramosca et al. [35] found a significant increase in triglycerides and esterified cholesterol in the liver of mice fed a diet containing 7.5\% OO for 8 weeks compared to mice fed a diet containing $7.5 \%$ corn oil. Some studies carried out in rats have shown that the consumption of OO leads to an increase in hepatic lipid deposition that is generally higher than with other diets, whether they are low in fatty acid $[36,37]$ or high in fatty acid $[38,39]$. The high richness of OO in MUFA, $60-70 \%$ [40], could explain the significant increase in hepatic triglycerides and esterified cholesterol induced by the olive oil diet. These results show that a high intake of OO favors a fat accumulation in the liver.

3.2. Effects of Diets on Mitochondrial Membrane. The study of membrane potential, ROS production, phospholipid, and fatty acid composition of the membrane made it possible to assess the effects of diets on mitochondrial membrane. No significant difference was observed between the diets concerning membrane potential and ROS production (Table 2).

Total phospholipid content in rat liver mitochondria was not modified whatever the diet. Refined PO promoted a significant $(p=0.016) \quad 10 \%$ decrease in membrane CL content compared to the control diet. Regarding the content of other membrane PL, no significant difference was observed between the diets (Table 3 ).

Mitochondrial membrane PL content found in control rats was consistent with many studies [33, 41]. PC and PE are the two main classes of membrane phospholipids and account for about $80 \%$ of total liver phospholipids. CL, a mitochondria-specific phospholipid [42], accounts for about $9 \%$ of total phospholipids in mitochondria homogenate. PC and PE play a key role in membrane function. PC tends to fluidise the membrane, while PE tends to stiffen it [33]. Therefore, the PC/ $\mathrm{PE}$ ratio is a key regulator of membrane integrity/fluidity [43]. A decrease in this ratio alters the properties of the membrane and could therefore also alter mitochondrial function.

Contrary to many studies $[25,41,44]$ that showed that HFD led to a decrease in the PC/PE ratio with an increase in CL content, no HFD in our study favored the decrease in PC/ $\mathrm{PE}$ ratio and the increase in CL. According to these authors, the increase in CL would be a means of compensating for the decrease in the $\mathrm{PC} / \mathrm{PE}$ ratio in order to maintain the properties of the membrane. Indeed, CL plays an important role in the stability of the physical properties of the membrane and mitochondrial cristae [45].

Despite the significant $10 \%$ decrease in CL content induced by refined PO, no HFD promoted increased ROS production or disruption of membrane potential. On the other hand, HFD in our study promoted a nonsignificant increase in the CP/PE ratio of $7.74 \%$ for refined PO, $7.14 \%$ for crude PO, 5.95\% for OO, and $4.17 \%$ for lard (Table 3). In addition, all HFD promoted a significant $(p<0.001)$ decrease in $\Delta 9$-desaturase activity compared to the control (Table 4). These results suggest an absence of deleterious effects of HFD based on palm oil (crude or refined), olive oil, and lard on the physicochemical properties of mitochondrial membrane [41, 46]. 

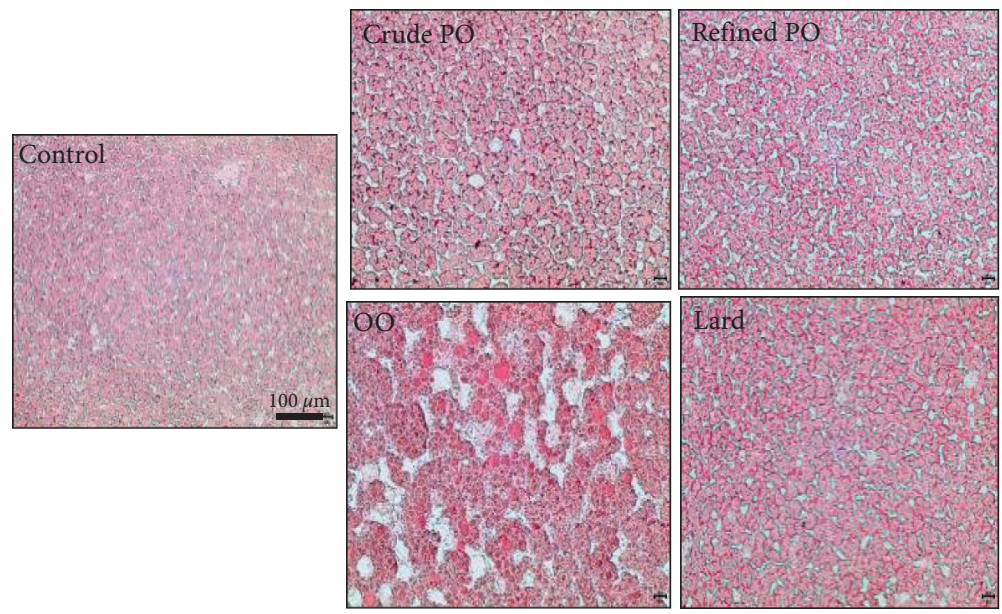

(a)

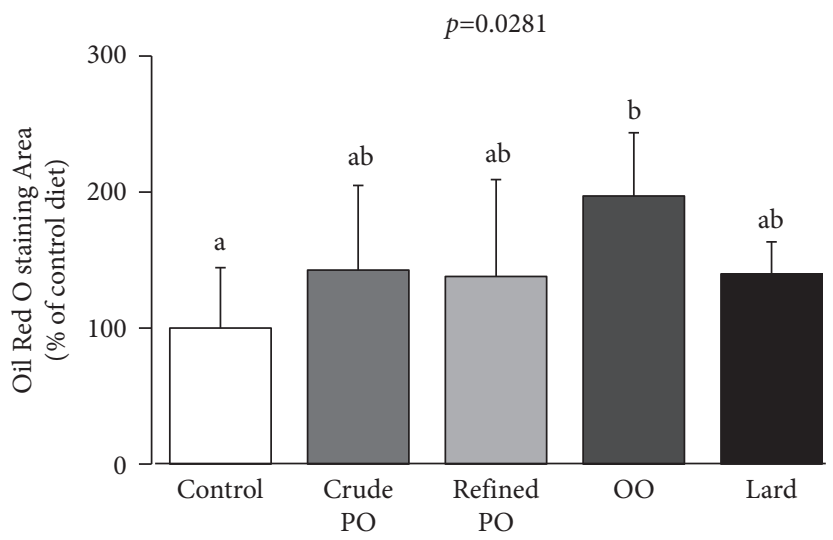

(b)

Figure 2: Effect of high dietary intake of palm oil, olive oil, and lard on hepatic lipid accumulation and steatosis. (a) Liver histology sections from a representative rat of each group, magnification 200x; (b) histograms represent liver lipid area quantification. The results are expressed as mean values $\pm \mathrm{SD}, n=7-8$ animals per group. The limit of statistical significance was set at $p<0.05$. Bar graphs with different letters (a, $b$, and c) are significantly different. OO, olive oil; PO, palm oil.

TABLe 2: Effect of diets on liver mitochondrial membrane potential and ROS production.

\begin{tabular}{|c|c|c|c|c|c|c|}
\hline & Control & Crude PO & Refined PO & $\mathrm{OO}$ & Lard & $p$ \\
\hline \multicolumn{7}{|c|}{ Mitochondrial membrane potential } \\
\hline Mitochondria (AU) & $0.51 \pm 0.3$ & $0.49 \pm 0.3$ & $0.53 \pm 0.1$ & $0.53 \pm 0.3$ & $0.53 \pm 0.3$ & NS \\
\hline Mitochondria + substrate (AU) & $0.68 \pm 0.1$ & $0.66 \pm 0.2$ & $0.70 \pm 0.1$ & $0.69 \pm 0.3$ & $0.68 \pm 0.2$ & NS \\
\hline Mitochondria + substrate + ADP (AU) & $0.52 \pm 0.1$ & $0.52 \pm 0.1$ & $0.53 \pm 0.1$ & $0.52 \pm 0.1$ & $0.51 \pm 0.1$ & NS \\
\hline \multicolumn{7}{|c|}{ Mitochondrial ROS production } \\
\hline Mitochondria (AU) & $95 \pm 20$ & $101 \pm 34$ & $100 \pm 28$ & $87 \pm 27$ & $103 \pm 34$ & NS \\
\hline Mitochondria + substrate (AU) & $150 \pm 23$ & $161 \pm 33$ & $159 \pm 32$ & $147 \pm 29$ & $152 \pm 32$ & NS \\
\hline Mitochondria + substrate + ADP (AU) & $141 \pm 24$ & $153 \pm 41$ & $154 \pm 37$ & $138 \pm 2$ & $141 \pm 33$ & NS \\
\hline
\end{tabular}

Values are expressed as mean $\pm \mathrm{SD}(n=7-8)$. ADP, adenosine diphosphate; AU, arbitrary units; OO, olive oil; PO, palm oil, ROS, reactive oxygen species.

In addition to the polar parts of PL, the fatty acid composition of PL also influences membrane properties. Thus, fatty acid composition of PL plays an important role in membrane fluidity due to the chain length, degree, and type of unsaturation of these fatty acids. More specifically, the SFA/MUFA ratio is inversely proportional to membrane fluidity [33].

A significant decrease $(p<0.01)$ was observed in the SFA/MUFA ratio compared to control diet with crude PO
(-23.07\%), refined PO $(-32.82 \%)$, and OO $(-55.38 \%)$ diets (Table 4).

These results show the beneficial effect of these three oils on membrane fluidity. Crude PO and refined PO diets promoted a significant $(p<0.01)$ decrease in the PUFA/SFA ratio compared to the control diet, by $40 \%$ and $42 \%$, respectively. This suggests that palm oil diets (crude or refined) may be more effective in protecting the mitochondrial 
TABLE 3: Effect of diets on liver mitochondrial membrane phospholipid (PL) composition.

\begin{tabular}{|c|c|c|c|c|c|c|}
\hline & Control & Crude PO & Refined PO & $\mathrm{OO}$ & Lard & $p$ \\
\hline Total PL (nmol/mg protein) & $228 \pm 9.1$ & $206 \pm 12$ & $236 \pm 13$ & $226 \pm 7.4$ & $230 \pm 11$ & NS \\
\hline LPC (\%) & $1.57 \pm 0.29$ & $1.74 \pm 0.19$ & $1.25 \pm 0.18$ & $1.43 \pm 0.25$ & $1.34 \pm 0.27$ & NS \\
\hline $\mathrm{SM}(\%)$ & $2.04 \pm 0.06$ & $1.89 \pm 0.10$ & $1.99 \pm 0.13$ & $2.16 \pm 0.15$ & $2.02 \pm 0.19$ & NS \\
\hline PC (\%) & $50.6 \pm 0.56$ & $51.9 \pm 0.62$ & $52.6 \pm 0.59$ & $51.8 \pm 0.65$ & $51.0 \pm 0.63$ & NS \\
\hline PI (\%) & $4.96 \pm 0.17$ & $5.54 \pm 0.22$ & $4.94 \pm 0.36$ & $5.00 \pm 0.27$ & $5.52 \pm 0.32$ & NS \\
\hline PS (\%) & $1.59 \pm 0.21$ & $1.20 \pm 0.21$ & $1.12 \pm 0.26$ & $1.15 \pm 0.27$ & $1.26 \pm 0.28$ & NS \\
\hline $\mathrm{PE}(\%)$ & $30.19 \pm 0.32$ & $28.88 \pm 0.55$ & $29.06 \pm 0.47$ & $29.30 \pm 0.52$ & $28.28 \pm 0.55$ & NS \\
\hline CL (\%) & $9.02 \pm 0.26 \mathrm{a}$ & $8.89 \pm 0.29 a$ & $8.04 \pm 0.32 b$ & $9.14 \pm 0.26 \mathrm{a}$ & $9.56 \pm 0.32 \mathrm{a}$ & 0.016 \\
\hline $\mathrm{PC} / \mathrm{PE}$ & $1.68 \pm 0.03$ & $1.80 \pm 0.06$ & $1.81 \pm 0.05$ & $1.78 \pm 0.05$ & $1.75 \pm 0.05$ & NS \\
\hline
\end{tabular}

Values are expressed as mean $\pm \mathrm{SD}(n=7-8)$. On the same line, values with different letters are significantly different. (\%), percentages in phosphorus of total phospholipids; CL, cardiolipin; LPC, lysophosphatidylcholine; OO, olive oil; PC, phosphatidylcholine; PE, phosphatidylethanolamine; PI, phosphatidylinositol; PL, phospholipid; PO, palm oil; PS, phosphatidylserine; SM, sphingomyelin.

TABLE 4: Effect of diets on liver mitochondrial phospholipid (PL) fatty acid (FA).

\begin{tabular}{|c|c|c|c|c|c|c|}
\hline & Control & Crude PO & Refined PO & $\mathrm{OO}$ & Lard & $p$ \\
\hline Total SFA & $39.1 \pm 0.43 a$ & $41.1 \pm 0.58 \mathrm{a}$ & $39.9 \pm 0.64 a$ & $30.9 \pm 1.33 b$ & $38.8 \pm 0.74 a$ & $<0.01$ \\
\hline C16:0 & $25.4 \pm 0.76 a$ & $27.4 \pm 0.65 b$ & $27.3 \pm 0.28 b$ & $20.4 \pm 0.81 c$ & $22.6 \pm 0.47 c$ & $<0.01$ \\
\hline $\mathrm{C} 18: 0$ & $12.5 \pm 0.75 \mathrm{ac}$ & $12.8 \pm 0.87 \mathrm{ac}$ & $11.6 \pm 0.78 \mathrm{ab}$ & $9.70 \pm 1.34 \mathrm{~b}$ & $15.3 \pm 1.00 \mathrm{c}$ & $<0.01$ \\
\hline Total MUFA & $20.5 \pm 1.22 \mathrm{a}$ & $28.4 \pm 1.73 b d$ & $30.8 \pm 1.17 b$ & $37.9 \pm 2.72 c$ & $24.7 \pm 1.79 \mathrm{ad}$ & $<0.01$ \\
\hline C16: $1 n-7$ & $3.40 \pm 0.35 a$ & $0.98 \pm 0.17 b$ & $1.11 \pm 0.18 \mathrm{~b}$ & $1.11 \pm 0.13 b$ & $0.89 \pm 0.07 b$ & $<0.01$ \\
\hline C18: $1 n-9$ & $13.4 \pm 0.92 \mathrm{a}$ & $25.0 \pm 1.69 \mathrm{bd}$ & $27.1 \pm 1.19 b$ & $33.3 \pm 2.64 c$ & $20.8 \pm 1.78 d$ & $<0.01$ \\
\hline C18: $1 n-7$ & $3.10 \pm 0.10 \mathrm{a}$ & $1.62 \pm 0.04 b$ & $1.60 \pm 0.09 b$ & $2.64 \pm 0.05 c$ & $2.50 \pm 0.10 \mathrm{c}$ & $<0.01$ \\
\hline Total PUFA & $40.4 \pm 1.29 a$ & $30.5 \pm 1.54 b$ & $29.3 \pm 0.66 b$ & $31.2 \pm 1.73 b$ & $36.5 \pm 1.14 \mathrm{a}$ & $<0.01$ \\
\hline C18: $2 n-6$ & $18.4 \pm 0.59 a$ & $11.1 \pm 0.80 b$ & $10.8 \pm 0.63 b$ & $13.5 \pm 0.80 c$ & $14.4 \pm 0.53 c$ & $<0.01$ \\
\hline C18: $3 n-6$ & $0.42 \pm 0.048 \mathrm{a}$ & $0.21 \pm 0.012 b$ & $0.21 \pm 0.011 b$ & $0.24 \pm 0.027 b$ & $0.21 \pm 0.021 b$ & $<0.01$ \\
\hline C18: $3 n-3$ & $0.78 \pm 0.058 \mathrm{a}$ & $0.14 \pm 0.020 \mathrm{~b}$ & $0.14 \pm 0.017 b$ & $0.24 \pm 0.031 b$ & $0.38 \pm 0.035 c$ & $<0.01$ \\
\hline C20: $3 n-6$ & $0.29 \pm 0.030$ & $0.26 \pm 0.051$ & $0.32 \pm 0.033$ & $0.32 \pm 0.042$ & $0.33 \pm 0.049$ & NS \\
\hline C20: $4 n-6$ & $15.1 \pm 1.07$ & $14.3 \pm 1.27$ & $13.3 \pm 0.68$ & $12.7 \pm 1.67$ & $15.7 \pm 1.20$ & NS \\
\hline C20: $5 n-3=\mathrm{EPA}$ & $0.23 \pm 0.034 \mathrm{a}$ & $0.04 \pm 0.006 b c$ & $0.03 \pm 0.006 b$ & $0.05 \pm 0.002 b c$ & $0.09 \pm 0.006 c$ & $<0.01$ \\
\hline $\mathrm{C} 22: 5 n-3$ & $0.36 \pm 0.023 a$ & $0.22 \pm 0.021 b$ & $0.23 \pm 0.014 b$ & $0.22 \pm 0.014 b$ & $0.41 \pm 0.047 \mathrm{a}$ & $<0.01$ \\
\hline C22: $6 n-3=\mathrm{DHA}$ & $4.19 \pm 0.31 \mathrm{a}$ & $2.74 \pm 0.18 b$ & $2.64 \pm 0.22 b$ & $3.02 \pm 0.42 b$ & $3.15 \pm 0.28 b$ & $<0.01$ \\
\hline Total $n-6$ & $34.6 \pm 1.03 a$ & $27.3 \pm 1.41 b$ & $26.2 \pm 0.57 b$ & $27.6 \pm 1.35 b$ & $31.4 \pm 0.90 c$ & $<0.01$ \\
\hline Total $n-3$ & $5.72 \pm 0.29 a$ & $3.19 \pm 0.18 b$ & $3.09 \pm 0.21 b$ & $3.57 \pm 0.41 b$ & $5.13 \pm 0.29 a$ & $<0.01$ \\
\hline$n-6 / n-3$ & $6.12 \pm 0.20 \mathrm{a}$ & $8.61 \pm 0.36 b$ & $8.66 \pm 0.53 b$ & $8.20 \pm 0.68 b$ & $6.18 \pm 0.22 a$ & $<0.01$ \\
\hline SFA/MUFA & $1.95 \pm 0.12 \mathrm{a}$ & $1.50 \pm 0.12 b$ & $1.31 \pm 0.08 \mathrm{~b}$ & $0.87 \pm 0.11 c$ & $1.64 \pm 0.16 \mathrm{ab}$ & $<0.01$ \\
\hline PUFA/SFA & $1.04 \pm 0.04 a$ & $0.74 \pm 0.04 b$ & $0.73 \pm 0.01 b$ & $1.01 \pm 0.04 \mathrm{a}$ & $0.94 \pm 0.02 \mathrm{a}$ & $<0.01$ \\
\hline$n$-3/PUFA & $0.141 \pm 0.004 \mathrm{a}$ & $0.105 \pm 0.004 b$ & $0.106 \pm 0.006 b$ & $0.113 \pm 0.007 \mathrm{~b}$ & $0.140 \pm 0.005 a$ & $<0.01$ \\
\hline PL UI & $152 \pm 4.56 \mathrm{a}$ & $133 \pm 4.68 b c$ & $131 \pm 2.19 b c$ & $141 \pm 5.92 \mathrm{ac}$ & $149 \pm 3.88 \mathrm{a}$ & $<0.01$ \\
\hline$\Delta 9$-desaturase & $0.132 \pm 0.010 \mathrm{a}$ & $0.035 \pm 0.005 b$ & $0.041 \pm 0.007 b$ & $0.054 \pm 0.005 b$ & $0.040 \pm 0.003 b$ & $<0.001$ \\
\hline$\Delta 6$-desaturase & $0.023 \pm 0.002 \mathrm{a}$ & $0.019 \pm 0.002 \mathrm{ab}$ & $0.020 \pm 0.002 \mathrm{a}$ & $0.018 \pm 0.002 \mathrm{ab}$ & $0.014 \pm 0.001 b$ & 0.045 \\
\hline$\Delta 5$-desaturase & $51.9 \pm 2.35$ & $52.8 \pm 4.61$ & $43.8 \pm 4.29$ & $41.4 \pm 4.93$ & $50.8 \pm 4.53$ & NS \\
\hline
\end{tabular}

Values are expressed as mean $\pm \mathrm{SD}(n=7-8)$. On the same line, values with different letters are significantly different. Major fatty acids are represented, and the results are expressed as percentage of fatty acid of total phospholipids. DHA, docosahexaenoic acid; EPA, eicosapentaenoic acid; MUFA, monounsaturated fatty acids; OO, olive oil; PL, phospholipids; PO, palm oil; PUFA, polyunsaturated fatty acids; SFA, saturated fatty acids; UI, unsaturation index. $\Delta 9$ desaturase $=16: 1 n-7 / 16: 0 ; \Delta 6$-desaturase $=18: 3 n-6 / 18: 2 n-6 ; \Delta 5$-desaturase $=20: 4 n-6 / 20: 3 n-6$. SFA/MUFA: SFA $=\%$ C16:0 $+\%$ C18:0 and MUFA $=\%$ $\mathrm{C} 16: 1+\% \mathrm{C} 18: 1$. PUFA/SFA: SFA $=\% \mathrm{C} 16: 0+\% \mathrm{C} 18: 0$ and PUFA $=\%$ all PUFA. $n-3 /$ PUFA: total $(n-3) /$ all PUFA.

membrane from ROS than other diets, as a decrease in the PUFA/SFA ratio makes mitochondria less susceptible to oxidative stress and lipid peroxidation [47].

$\Delta 6$-desaturase catalyzes the initial desaturation (limiting step) of linoleic and a-linolenic acids. Through a sequence of desaturation and chain lengthening reactions, these two essential fatty acids form arachidonic, eicosapentaenoic, and docosahexaenoic acids, which are the main PUFA substituents of membrane phospholipids, which regulate cell membrane fluidity [48]. The significant decrease $(p=0.045)$ of $\Delta 6$-desaturase activity in the lard diet compared to the control diet (Table 4) could lead to a long-term disturbance of membrane fluidity in the mitochondria of lard-fed rats due to the absence of arachidonic, eicosapentaenoic, and docosahexaenoic acids in the phospholipids of the mitochondrial membranes [48, 49].

As shown in Table 4, despite their high SFA content, PO and lard diets did not significantly increase SFA content of the mitochondrial membrane PL. On the other hand, all HFD favored, compared to the control diet, an increase in MUFA and a decrease in PUFA of the mitochondrial membrane PL. These variations were significant with crude 
TABLE 5: Variation in mitochondrial enzyme activity.

\begin{tabular}{|c|c|c|c|c|c|c|}
\hline & Control & Crude PO & Refined PO & $\mathrm{OO}$ & Lard & $p$ \\
\hline Complex I (mU/mg protein) & $1.56 \pm 0.25$ & $1.31 \pm 0.16$ & $1.40 \pm 0.14$ & $1.41 \pm 0.14$ & $1.42 \pm 0.11$ & NS \\
\hline Complex II (mU/mg protein) & $1.85 \pm 0.05^{\mathrm{a}}$ & $1.74 \pm 0.04^{\mathrm{ab}}$ & $1.80 \pm 0.08^{\mathrm{a}}$ & $1.57 \pm 0.07^{\mathrm{b}}$ & $1.70 \pm 0.07^{\mathrm{ab}}$ & 0.028 \\
\hline Complex II + III (mU/mg protein) & $0.078 \pm 0.006$ & $0.077 \pm 0.005$ & $0.094 \pm 0.010$ & $0.065 \pm 0.007$ & $0.069 \pm 0.007$ & NS \\
\hline Complex IV (mU/mg protein) & $0.796 \pm 0.086$ & $0.767 \pm 0.090$ & $0.846 \pm 0.129$ & $0.819 \pm 0.097$ & $0.902 \pm 0.058$ & NS \\
\hline Citrate synthase (mU/mg protein) & $96.9 \pm 6.12$ & $103 \pm 7.12$ & $101 \pm 6.68$ & $106 \pm 5.12$ & $104 \pm 6.72$ & NS \\
\hline CLS (WB) & $1.00 \pm 0.22^{\mathrm{a}}$ & $0.64 \pm 0.84^{\mathrm{a}}$ & $0.63 \pm 0.90^{\mathrm{a}}$ & $0.52 \pm 0.12^{\mathrm{b}}$ & $0.38 \pm 0.84^{\mathrm{b}}$ & 0.023 \\
\hline
\end{tabular}

Values are expressed as mean \pm SD $(n=7-8)$. On the same line, values with different letters are significantly different. CLS, cardiolipin synthase; WB, western blot.

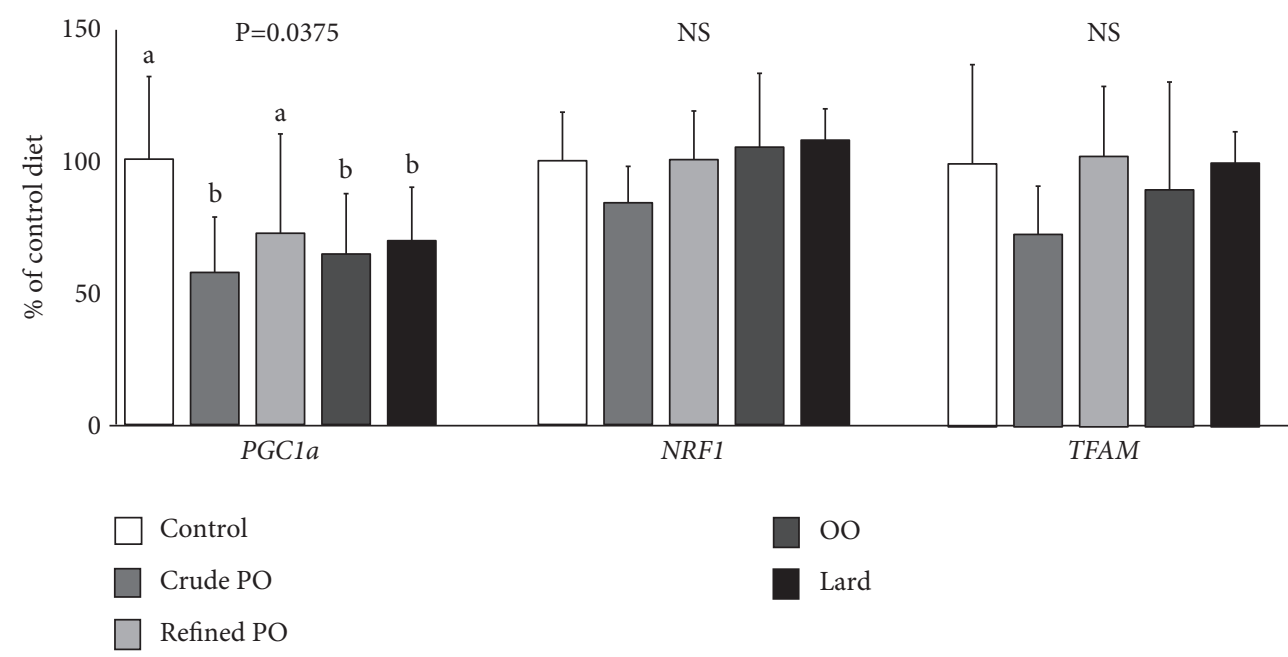

Figure 3: Expression of genes involved in liver mitochondrial biogenesis. Values are expressed as mean \pm SD $(n=7-8)$. Histogram bars with different letters are significantly different. PO, palm oil; OO, olive oil; Pgcl $\alpha$, peroxisome proliferator activator receptor $\gamma$ coactivator-1 $\alpha$; Nrf1, nuclear respiratory factor 1; Tfam, mitochondrial transcription factor A.

PO, refined PO, and OO but not with lard (Table 4). EPA and DHA contents of mitochondrial membrane PL were significantly lower in all HFD compared with the control diet (Table 4). This change in the FA composition of mitochondrial membrane PL has been observed by other authors $[23,25,50]$. These results show that excess crude PO, refined $\mathrm{PO}$, OO, and lard alters the composition of the mitochondrial membrane.

3.3. Effects of Diet on Mitochondrial Enzyme Activity. The diets caused disturbances in the activity of certain mitochondrial enzymes (Table 5). OO and lard diets significantly $(p=0.023)$ reduced hepatic CLS protein expression by $48 \%$ and $62 \%$, respectively, compared to the control diet. Complex II activity was significantly $(p=0.028)$ decreased by $15 \%$ in OO diet compared to the control diet (Table 5 ).

CLS is an essential enzyme in the biosynthesis of CL [51]. Decreasing CLS protein expression could lead to a decrease in CL synthesis. CL is closely associated with mitochondrial proteins and is thought to be involved in their function. Indeed, CL is required for optimal activity of complex I $[52,53]$, complex III $[52,54]$, complex IV [55], and complex $\mathrm{V}[56]$.

The significant $10 \%$ decrease in mitochondrial membrane CL content induced by refined PO did not affect the activity of respiratory chain complex. On the contrary, a significant decrease in complex II activity with $\mathrm{OO}$ diet associated with decrease of CLS protein expression was observed.

These results show that palm oil diets (crude or refined) do not have deleterious effects on respiratory chain enzymes as no significant differences were observed between control diet and PO diets with respect to their effects on citrate synthase, CLS, and respiratory chain complex. Lard had a deleterious effect on CLS protein expression, while OO had a deleterious effect on respiratory chain complex II activity and CLS protein expression.

3.4. Effects of Diets on Mitochondrial Biogenesis. No significant differences were observed between the diets concerning the gene expression of Nrf1 and Tfam. In contrast, all HFD repressed Pgcl $\alpha$ gene expression. This decrease in $\operatorname{Pgc} 1 \alpha$ gene expression was significant $(p=0.0375)$ with crude $\mathrm{PO}, \mathrm{OO}$, and lard diets compared to the control diet (Figure 3).

Many authors [57, 58] have shown that excessive fat intake promotes the decrease of $\operatorname{Pgc} 1 \alpha$ gene expression.

$\operatorname{Pgc} 1 \alpha$ is the main regulator of mitochondrial biogenesis, activating the expression of respiratory chain subunits and Nrf1, which is a potent stimulator of the expression of Tfam, polymerase RNA mitochondrial (Polrmt), and mitochondrial transcription factor $\mathrm{B} 1$ and $\mathrm{B} 2$ (Tfb1m and $\mathrm{Tfb} 2 \mathrm{~m}$ ), 
which are factors involved in mitochondrial DNA replication and transcription [59, 60]. As such, a decrease in Pgcl $\alpha$ gene expression will result in a long-term disruption of mitochondrial function.

Although no significant differences in Nrfland Tfam expression were observed between diets, the decrease in $\operatorname{Pgc} 1 \alpha$ gene expression by HFD indicates that these diets impaired mitochondrial biogenesis.

Clearly, further work examining the metabolic role of excess vegetable oil in various pathological and nonpathological conditions/models, including more parameters, is warranted to learn more. Nevertheless, this study showed that $\mathrm{PO}$ and $\mathrm{OO}$ improved the fluidity of liver mitochondrial membrane. On the other hand, OO favored a fat accumulation in the liver and disturbed the activity of some enzymes of the respiratory chain. It appears that the increased intake of dietary fat, whatever its composition in saturated and unsaturated fatty acids, favored a disturbance of mitochondrial biogenesis. In light of these results, the normocaloric diet could help in the prevention of certain diseases related to mitochondrial dysfunction.

\section{Abbreviations}

AU: Arbitrary unit

CL: Cardiolipin

CLS: Cardiolipin synthase

CS: Citrate synthase

HFD: High-fat diet

IR: Insulin resistance

OO: Olive oil

PL: Phospholipid

PO: Palm oil

ROS: Reactive oxygen species

UI: Unsaturation index

WB: Western blot

WW: Wet weight.

\section{Data Availability}

The data used to support the findings of this study are included within the article.

\section{Ethical Approval}

All procedures were authorized by the academic ethics committee of the University of Montpellier. The experiment was carried out in strict accordance with the guidelines for the management and use of laboratory animals. All the laboratory procedures were used to reduce the pain of the rats.

\section{Conflicts of Interest}

The authors declare that they have no conflicts of interest regarding the publication of this paper.

\section{Acknowledgments}

This work was supported by a research grant from SANIA (No. 2015/UM/150666).

\section{References}

[1] H. M. McBride, M. Neuspiel, and S. Wasiak, "Mitochondria: more than just a powerhouse," Current Biology: $C B$, vol. 16, pp. R551-R560, 2006.

[2] T. Valero, "Editorial (thematic issue: mitochondrial biogenesis: pharmacological approaches)," Current Pharmaceutical Design, vol. 20, no. 35, pp. 5507-5509, 2014.

[3] F. Sanchis-Gomar, J. Garcia-Gimenez, M. Gomez-Cabrera, and F. Pallardo, "Mitochondrial biogenesis in health and disease. Molecular and therapeutic approaches," Current Pharmaceutical Design, vol. 20, no. 35, pp. 5619-5633, 2014.

[4] D. L. Johannsen and E. Ravussin, "The role of mitochondria in health and disease," Current Opinion in Pharmacology, vol. 9, no. 6, pp. 780-786, 2009.

[5] P. J. Quinn, "The fluidity of cell membranes and its regulation," Progress in Biophysics and Molecular Biology, vol. 38, pp. 1-104, 1981.

[6] A. J. Hulbert, N. Turner, L. H. Storlien, and P. L. Else, "Dietary fats and membrane function: implications for metabolism and disease," Biological Reviews, vol. 80, no. 1, pp. 155-169, 2005.

[7] K. Morino, K. F. Petersen, S. Dufour et al., "Reduced mitochondrial density and increased IRS-1 serine phosphorylation in muscle of insulin-resistant offspring of type 2 diabetic parents," Journal of Clinical Investigation, vol. 115, no. 12, pp. 3587-3593, 2005.

[8] M. A. Abdul-Ghani and R. A. DeFronzo, "Pathogenesis of insulin resistance in skeletal muscle," Journal of Biomedicine and Biotechnology, vol. 2010, Article ID 476279, 19 pages, 2010.

[9] V. T. Samuel, K. F. Petersen, and G. I. Shulman, "Lipid-induced insulin resistance: unravelling the mechanism," The Lancet, vol. 375, no. 9733, pp. 2267-2277, 2010.

[10] L. Liu, S. Yu, R. S. Khan et al., "DGAT1 deficiency decreases PPAR expression and does not lead to lipotoxicity in cardiac and skeletal muscle," Journal of Lipid Research, vol. 52, no. 4, pp. 732-744, 2011.

[11] P. Jiao, J. Ma, B. Feng et al., "FFA-induced adipocyte inflammation and insulin resistance: involvement of ER stress and IKK $\beta$ pathways," Obesity, vol. 19, no. 3, pp. 483-491, 2011.

[12] L. Simon-Szabó, M. Kokas, J. Mandl, G. Kéri, and M. Csala, "Metformin attenuates palmitate-induced endoplasmic reticulum stress, serine phosphorylation of IRS-1 and apoptosis in rat insulinoma cells," PLoS One, vol. 9, no. 6, Article ID e97868, 2014.

[13] K. Sundram, R. Sambanthamurthi, and Y. A. Tan, "Palm fruit chemistry and nutrition," Asia Pacific Journal of Clinical Nutrition, vol. 12, pp. 355-362, 2003.

[14] A. A. Mondé, F. Michel, M. A. Carbonneau et al., "Comparative study of fatty acid composition, vitamin $\mathrm{E}$ and carotenoid contents of palm oils from four varieties of oil palm from Côte d'Ivoire," Journal of the Science of Food and Agriculture, vol. 89, pp. 2535-2540, 2009.

[15] A. Rohman, K. Triyana, S. Sismindari, and Y. Erwanto, "Differentiation of lard and other animal fats based on triacylglycerols composition and principal component analysis," International Food Research Journal, vol. 19, pp. 475-479, 2012. 
[16] M. Haddam, H. Chimi, and A. Amine, "Formulation d'une huile d'olive de bonne qualité," Oilseeds and fats, Crops and Lipids, vol. 21, no. 5, Article ID D507, 2014.

[17] R. W. Owen, W. Mier, A. Giacosa, W. E. Hull, B. Spiegelhalder, and H. Bartsch, "Phenolic compounds and squalene in olive oils: the concentration and antioxidant potential of total phenols, simple phenols, secoiridoids, lignansand squalene," Food and Chemical Toxicology, vol. 38, no. 8, pp. 647-659, 2000.

[18] F. Perez-Jimenez, G. Alvarez de Cienfuegos, and L. Badimon, "International conference on the healthy effect of virgin olive oil," European Journal of Clinical Investigation, vol. 35, pp. 421-424, 2005.

[19] M.-I. Covas, V. Ruiz-Gutiérrez, R. De la Torre et al., "Minor components of olive oil: evidence to date of health benefits in humans," Nutrition Reviews, vol. 64, no. 10, pp. 20-30, 2006.

[20] Y. F. Djohan, E. Badia, B. Bonafos et al., "High dietary intake of palm oils compromises glucose tolerance whereas high dietary intake of olive oil compromises liver lipid metabolism and integrity," European Journal of Nutrition, vol. 58, no. 8, pp. 3091-3107, 2019.

[21] G. Fouret, S. Gaillet, J. Lecomte et al., "20-Week follow-up of hepatic steatosis installation and liver mitochondrial structure and activity and their interrelation in rats fed a high-fat-highfructose diet," British Journal of Nutrition, vol. 119, no. 4, pp. 368-380, 2018.

[22] M. M. Bradford, "A rapid and sensitive method for the quantitation of microgram quantities of protein utilizing the principle of protein-dye binding," Analytical Biochemistry, vol. 72, no. 1-2, pp. 248-254, 1976.

[23] M. Aoun, C. Feillet-Coudray, G. Fouret et al., "Rat liver mitochondrial membrane characteristics and mitochondrial functions are more profoundly altered by dietary lipid quantity than by dietary lipid quality: effect of different nutritional lipid patterns," British Journal of Nutrition, vol. 107, no. 5, pp. 647-659, 2012.

[24] G. Barja, "The quantitative measurement of $\mathrm{H}_{2} \mathrm{O}_{2}$ generation in isolated mitochondria," Journal of Bioenergetics and Biomembranes, vol. 34, no. 3, pp. 227-233, 2002.

[25] M. Aoun, G. Fouret, F. Michel et al., "Dietary fatty acids modulate liver mitochondrial cardiolipin content and its fatty acid composition in rats with non alcoholic fatty liver disease," Journal of Bioenergetics and Biomembranes, vol. 44, no. 4, pp. 439-452, 2012.

[26] A. J. Janssen, F. J. Trijbels, R. C. Sengers et al., "Spectrophotometric assay for complex I of the respiratory chain in tissue samples and cultured fibroblasts," Clinical Chemistry, vol. 53, no. 4, pp. 729-734, 2007.

[27] P. Rustin, D. Chretien, T. Bourgeron et al., "Biochemical and molecular investigations in respiratory chain deficiencies," Clinica Chimica Acta, vol. 228, no. 1, pp. 35-51, 1994.

[28] D. C. Wharton and A. Tzagoloff, "[45] Cytochrome oxidase from beef heart mitochondria," Methods in Enzymology, vol. 10, pp. 245-250, 1967.

[29] J. Folch, M. Lees, and G. H. S. Stanley, “A simple method for the isolation and purification of total lipides from animal tissues," Journal of Biological Chemistry, vol. 226, no. 1, pp. 497-509, 1957.

[30] G. R. Bartlett, "Phosphorus assay in column chromatography," Journal of Biological Chemistry, vol. 234, no. 3, pp. 466-468, 1959.

[31] G. Lepage and C. C. Roy, "Direct transesterification of all classes of lipids in a one-step reaction," Journal of Lipid Research, vol. 27, no. 1, pp. 114-120, 1986.
[32] E. Warensjö, U. Risérus, I. B. Gustafsson, R. Mohsen, T. Cederholm, and B. Vessby, "Effects of saturated and unsaturated fatty acids on estimated desaturase activities during a controlled dietary intervention," Nutrition, Metabolism, and Cardiovascular Diseases: Nutrition, Metabolism, and Cardiovascular Diseases, vol. 18, pp. 683-690, 2008.

[33] V. Tepsic, V. Ristic, D. Ristic, N. Vasiljevic, and M. Pecelj-Gec, "Heart phospholipid content and fatty acid composition in the rat after feeding different lipid supplemented diets," Physiological Research, vol. 47, pp. 413-418, 1998.

[34] J. M. Arbones-Mainar, K. Ross, G. J. Rucklidge et al., "Extra virgin olive oils increase hepatic fat accumulation and hepatic antioxidant protein levels in APOE-/- mice," Journal of Proteome Research, vol. 6, no. 10, pp. 4041-4054, 2007.

[35] A. Ferramosca, V. Savy, and V. Zara, "Olive oil increases the hepatic triacylglycerol content in mice by a distinct influence on the synthesis and oxidation of fatty acids," Bioscience Biotechnology and Biochemistry, vol. 72, no. 1, pp. 62-69, 2008.

[36] V. Ruiz-Gutiérrez, C. M. Vázquez, and C. Santa-Maria, "Liver lipid composition and antioxidant enzyme activities of spontaneously hypertensive rats after ingestion of dietary fats (fish, olive and high-oleic sunflower oils)," Bioscience Reports, vol. 21, no. 3, pp. 271-285, 2001.

[37] J. S. Perona and V. Ruiz-Gutiérrez, "Effect of two high-oleic oils on the liver lipid composition of spontaneously hypertensive rats," Life Sciences, vol. 66, no. 6, pp. 521-531, 2000.

[38] M. P. Portillo, M. Chávarri, D. Durán, V. M. Rodriguez, and M. T. Macarulla, "Differential effects of diets that provide different lipid sources on hepatic lipogenic activities in rats under ad libitum or restricted feeding," Nutrition, vol. 17, no. 6, pp. 467-473, 2001.

[39] H. Takeuchi, T. Nakamoto, Y. Mori et al., "Comparative effects of dietary fat types on hepatic enzyme activities related to the synthesis and oxidation of fatty acid and to lipogenesis in rats," Bioscience Biotechnology and Biochemistry, vol. 65, no. 8, pp. 1748-1754, 2001.

[40] A. Montaño, M. Hernández, I. Garrido, J. L. Llerena, and F. Espinosa, "Fatty acid and phenolic compound concentrations in eight different monovarietal virgin olive oils from extremadura and the relationship with oxidative stability," International Journal of Molecular Sciences, vol. 17, no. 11, p. 1960, 2016.

[41] G. Fouret, E. Tolika, J. Lecomte et al., "The mitochondrialtargeted antioxidant, MitoQ, increases liver mitochondrial cardiolipin content in obesogenic diet-fed rats," Biochimica et Biophysica Acta (BBA)-Bioenergetics, vol. 1847, no. 10, pp. 1025-1035, 2015.

[42] N. Gebert, A. S. Joshi, S. Kutik et al., "Mitochondrial cardiolipin involved in outer-membrane protein biogenesis: implications for Barth syndrome," Current Biology, vol. 19, no. 24, pp. 2133-2139, 2009.

[43] B. M. Arendt, D. W. L. Ma, B. Simons et al., "Nonalcoholic fatty liver disease is associated with lower hepatic and erythrocyte ratios of phosphatidylcholine to phosphatidylethanolamine," Applied Physiology Nutrition and Metabolism, vol. 38, no. 3, pp. 334-340, 2013.

[44] I. G. Stavrovskaya, S. S. Bird, V. R. Marur et al., "Dietary macronutrients modulate the fatty acyl composition of rat liver mitochondrial cardiolipins," Journal of Lipid Research, vol. 54, no. 10, pp. 2623-2635, 2013.

[45] T. Ohtsuka, M. Nishijima, K. Suzuki, and Y. Akamatsu, "Mitochondrial dysfunction of a cultured Chinese hamster 
ovary cell mutant deficient in cardiolipin," Journal of Biological Chemistry, vol. 268, no. 30, pp. 22914-22919, 1993.

[46] G. Serviddio, F. Bellanti, and G. Vendemiale, "Free radical biology for medicine: learning from nonalcoholic fatty liver disease," Free Radical Biology and Medicine, vol. 65, pp. 952-968, 2013.

[47] M. J. Kang, M. S. Shin, J. N. Park, and S. S. Lee, "The effects of polyunsaturated:saturated fatty acids ratios and peroxidisability index values of dietary fats on serum lipid profiles and hepatic enzyme activities in rats," British Journal of Nutrition, vol. 94, no. 4, pp. 526-532, 2005.

[48] W. Stoffel, B. Holz, and B. Jenke, "Delta6-desaturase (FADS2) deficiency unveils the role of omega3- and omega6-polyunsaturated fatty acids," The EMBO Journal, vol. 27, no. 17, pp. 2281-2292, 2008.

[49] C. H. Le, C. M. Mulligan, M. A. Routh et al., "Delta-6desaturase links polyunsaturated fatty acid metabolism with phospholipid remodeling and disease progression in heart failure," Circulation. Heart failure, vol. 7, no. 1, pp. 172-183, 2014.

[50] E. A. Herbst, S. Paglialunga, C. Gerling et al., "Omega-3 supplementation alters mitochondrial membrane composition and respiration kinetics in human skeletal muscle," Journal of Physiology, vol. 592, no. 6, pp. 1341-1352, 2014.

[51] M. Schlame and K. Y. Hostetler, "Cardiolipin synthase from mammalian mitochondria," Biochimica et Biophysica Acta, vol. 1348, pp. 207-213, 1997.

[52] M. Fry and D. E. Green, "Cardiolipin requirement for electron transfer in complex I and III of the mitochondrial respiratory chain," Journal of Biological Chemistry, vol. 256, pp. 1874-1880, 1981.

[53] S. Dröse, K. Zwicker, and U. Brandt, "Full recovery of the NADH: ubiquinone activity of complex I (NADH: ubiquinone oxidoreductase) from Yarrowia lipolytica by the addition of phospholipids," Biochimica et Biophysica Acta, vol. 1556, pp. 65-72, 2002.

[54] C. Lange, J. H. Nett, B. L. Trumpower, and C. Hunte, "Specific roles of protein-phospholipid interactions in the yeast cytochrome bc1 complex structure," The EMBO Journal, vol. 20, pp. 6591-6600, 2001.

[55] N. C. Robinson, "Functional binding of cardiolipin to cytochrome c oxidase," Journal of Bioenergetics and Biomembranes, vol. 25, pp. 153-163, 1993.

[56] K. S. Eble, W. B. Coleman, R. R. Hantgan, and C. C. Cunningham, "Tightly associated cardiolipin in the bovine heart mitochondrial ATP synthase as analyzed by $31 \mathrm{P}$ nuclear magnetic resonance spectroscopy," Journal of Biological Chemistry, vol. 265, pp. 19434-19440, 1990.

[57] J. Hoeks, M. K. Hesselink, A. P. Russell et al., "Peroxisome proliferator-activated receptor-gamma coactivator-1 and insulin resistance: acute effect of fatty acids," Diabetologia, vol. 49, pp. 2419-2426, 2006.

[58] C. Feillet-Coudray, G. Fouret, R. Ebabe-Elle et al., "The mitochondrial-targeted antioxidant MitoQ ameliorates metabolic syndrome features in obesogenic diet-fed rats better than Apocynin or Allopurinol," Free Radical Research, vol. 48, pp. 1232-1246, 2014.

[59] R. C. Scarpulla, "Nuclear control of respiratory gene expression in mammalian cells," Journal of Cellular Biochemistry, vol. 97, pp. 673-683, 2006.

[60] C. Wang, Z. Li, Y. Lu et al., "Cyclin D1 repression of nuclear respiratory factor 1 integrates nuclear DNA synthesis and mitochondrial function," Proceedings of the National Academy of Sciences of USA, vol. 103, pp. 11567-11572, 2006. 